\title{
Isolated Hemifacial Sensory Impairment with Onion Skin Distribution Caused by Small Pontine Hemorrhage
}

\author{
N. Toratani H. Moriwaki B. Hyon H. Naritomi \\ Department of Cerebrovascular Medicine, National Cardiovascular Center, Osaka, Japan
}

\begin{abstract}
Dear Sir,
Isolated trigeminal sensory neuropathy due to pontine hemorrhage has only been found in a few previous cases [1-4]. In these, the hemorrhage affected either the principal sensory nucleus $[1,2]$ or the trigeminal nerve roots $[3,4]$ and produced facial sensory impairments along the ophthalmic (V1), maxillary (V2) and/or mandibular (V3) nerve dermatome. We report a patient with a small pontine hemorrhage that affected the upper part of the spinal trigeminal nuclei, mainly the nucleus oralis and interpolaris. She developed isolated facial tactile sensory impairment with 'onion skin' distribution in the absence of other neurological deficits. Onion skin type facial sensory impairment caused by these upper spinal trigeminal nuclei due to stroke has never been previously reported.
\end{abstract}

\section{Case Report}

A 61-year-old woman suddenly felt numbness around the right side of her lips while talking on the telephone. She experienced neither headache nor vomiting. She had a history of hypertension and hyperlipidemia but received no medical treatment. She was admitted to our hospital $1 \mathrm{~h}$ after onset. At the time of admission, her blood pressure was 200/114 mm $\mathrm{Hg}$, while the heart beats were 108 per minute and regular. Neurological examinations revealed an alert and well-oriented woman with isolated tactile sensory impairment over the right trigeminal distribution including the oral cavity. Pain and temperature sensations were not impaired. The hypesthesia showed so-called onion skin distribution and was least intense in the perioral and perinasal areas and most prominent in the most peripheral parts of the face, such as the forehead and chin. Corneal sensation and corneal reflex were well preserved. No weakness of the masseteric muscle or jaw deviation was observed. Taste sensation was normal. There was no abnormality in cranial nerves, coordination or body sensation, except for the face.

Brain CT performed on admission and $1.5 \mathrm{~h}$ after onset demonstrated a small, high-density area at the right pontine tegmentum (fig. 1). The diameter of the hematoma was less than $1 \mathrm{~cm}$ and did not enlarge on a second CT performed $6 \mathrm{~h}$ after onset. $\mathrm{T}_{1}$-weighted MRI on the seventh day showed a column-shaped hematoma in areas extending from the middle part to the lower portion of the pons and accompanied by a smaller sublesion (fig. 2A-C). The hematoma did not reach the medulla oblongata, as confirmed by coronal MRI. MR angiography on the seventh day disclosed no abnormality suggestive of arteriovenous malformation, aneurysm or cavernous angioma. The diagnosis of hypertensive pontine hemorrhage was established on the basis of such imaging studies. She was discharged on day 11, at which time her facial hypesthesia was mild in degree. Three months thereafter, her facial hypesthesia was confined to the areas around the right side of the lips.

\section{Discussion}

Pontine hemorrhage presenting a trigeminal neuropathy is usually associated with other cranial nerve deficiencies. Even in cases of small hematoma localized in the tegmentum, neurological examination usually reveals a variety of symptoms depending on the magnitude of basilar involvement, such as oculomotor abnormalities, ataxia, action tremor, ipsilateral miosis, hemiparesis or facial numbness [5]. Isolated trigeminal sensory neuropathy is seldom observed in cases of pontine hemorrhage. To the best of our knowledge, only 4 such cases have been reported previously [1-4] (table 1). In these 4 cases, the lesions affected the principal sensory nucleus or the trigeminal nerve roots, and all of the facial sensory impairments were observed along the V1, V2 or V3 dermatomes.

The trigeminal nerve contains both sensory and motor components. The ma-

\section{KARGER}

Fax +41 613061234

E-Mail karger@karger.ch

www.karger.com (c) 2008 S. Karger AG, Basel

0014-3022/08/0594-0192\$24.50/0

Accessible online at:

www.karger.com/ene
Naomi Toratani

Department of Cerebrovascular Medicine

National Cardiovascular Center

5-7-1 Fujishirodai, Suita, Osaka 565-8565 (Japan)

Tel. +81 66833 5012, Fax +81 66872 7486, E-Mail tora@hsp.ncvc.go.jp 
Fig. 1. Brain CT, obtained $1.5 \mathrm{~h}$ after onset of symptoms, discloses a small, high-density area (arrow) in the medial portion of the right pontine tegmentum.

Fig. 2. $T_{1}$-weighted $M R$ images obtained on the seventh day. A Transaxial image discloses hematoma at the pontine tegmentum, the diameter of which is smaller than $1 \mathrm{~cm}$. B, C The sagittal and coronal images demonstrate that the hematoma is column-shaped, extending from the middle pons to the lower pons and accompanied by a smaller sublesion.
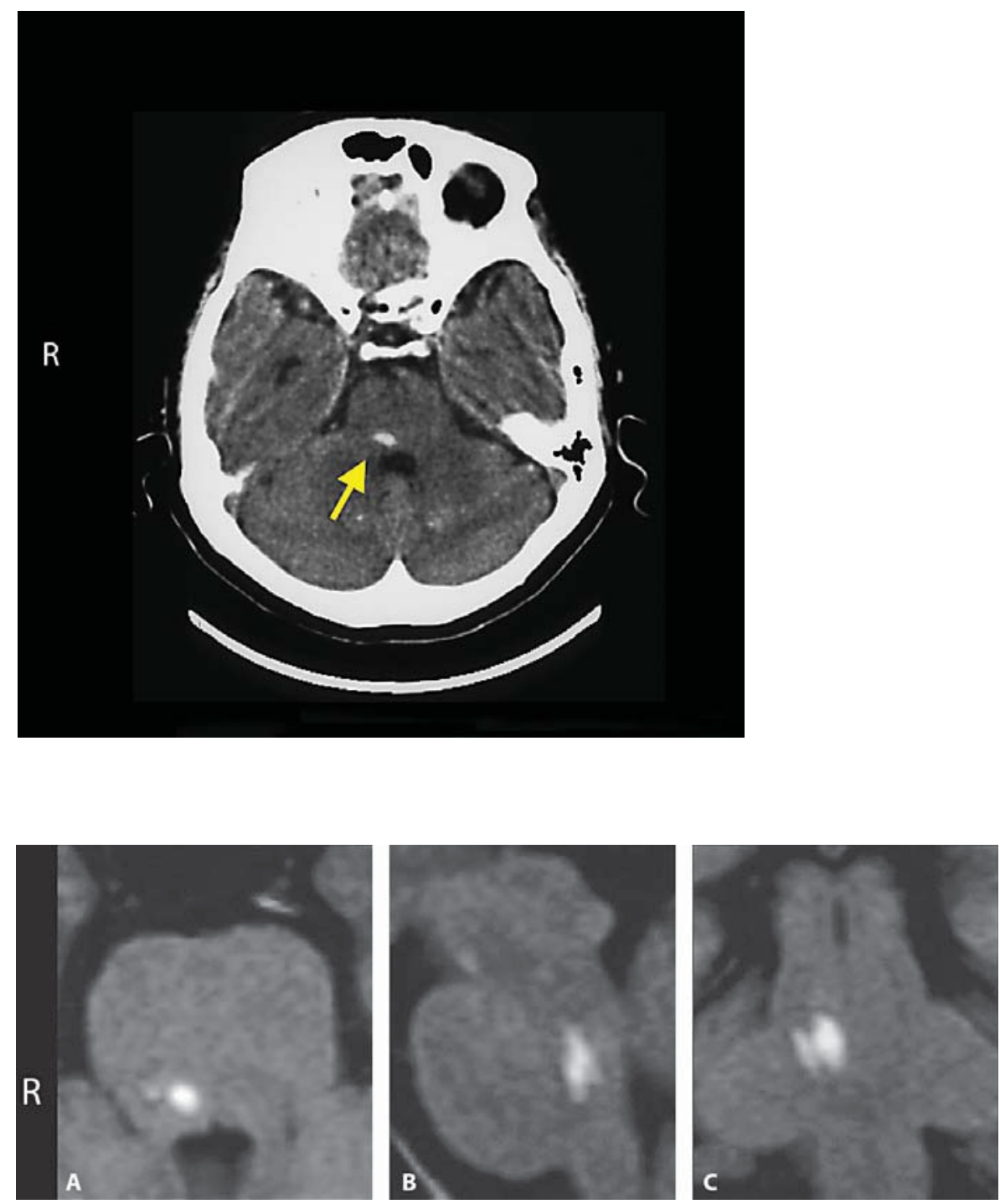

Table 1. Reported cases of pontine hemorrhage with isolated trigeminal sensory neuropathy

\begin{tabular}{|c|c|c|c|c|}
\hline Authors and year & $\begin{array}{l}\text { Age } \\
\text { years }\end{array}$ & Sex & Distribution & Location of the lesion \\
\hline Holtzman et al. [1], 1987 & 45 & M & $\mathrm{V} 2, \mathrm{~V} 3$ & trigeminal principal sensory nucleus \\
\hline Kim et al. [2], 1994 & 31 & M & $\mathrm{V} 1<\mathrm{V} 2, \mathrm{~V} 3$ & trigeminal nerve root and trigeminal principal sensory nucleus \\
\hline Komiyama et al. [3], 1993 & 49 & M & V1 & trigeminal nerve root \\
\hline Almeida et al. [4], 1999 & 62 & M & V1 & trigeminal nerve root \\
\hline The present case & 61 & $\mathrm{~F}$ & $\begin{array}{l}\mathrm{V} 1 \sim \mathrm{V} 3 \\
\text { 'onion skin' pattern }\end{array}$ & nucleus oralis or interpolaris of trigeminal spinal nucleus \\
\hline
\end{tabular}


jority of trigeminal sensory fibers conveying tactile sensation does not descend but enters the principal sensory nucleus. Other trigeminal sensory fibers carrying pain and temperature sensations descend in the trigeminal tract and enter the spinal trigeminal nucleus. The spinal trigeminal nucleus consists of 3 components - the nucleus oralis, nucleus interpolaris and nucleus caudalis - which are located along the longitudinal axis of the brain stem [68]. Among these nuclei, the fibers carrying pain and temperature sensations enter the nucleus caudalis, which is located at the most caudal part of the spinal trigeminal nucleus. The somatotopic representation of the face within the nucleus caudalis is in a segmental distribution similar to onion skin: the perioral areas of the face are represented by the rostral portion of the nucleus, and more outer areas of the face are represented by the caudal portion of the nucleus. Therefore, lesions affecting the nucleus caudalis at the medulla oblongata may cause onion skin type sensory impairment, as occasionally observed in cases of syringobulbia. On the other hand, the functions of the other 2 nuclei - the nucleus oralis and interpolaris - still remain unclear. Fibers carrying tactile sensation mainly enter the principal sensory nucleus and partly so the upper part of the spinal trigeminal nucleus - the nucleus oralis and interpolaris [9]. The tactile representation of the face within the nucleus oralis and interpolaris remains unclear.

In the present case, isolated tactile sensory impairment with onion skin distribution was observed in the hemifacial area ipisilateral to the side of the small pontine hemorrhage. According to the CT and MRI studies, the hematoma was located more medially to the principal sensory nucleus and affected the upper part of the spinal trigeminal nucleus, such as the nucleus oralis and interporalis. The onion skin type hypesthesia is unlikely to be caused by lesions of the principal sensory nucleus. The present case suggests that the onion skin type hypesthesia may be caused by lesions in the nucleus oralis or interpolaris. The somatotopic representation of the face in the nucleus oralis and interpolaris may be in a segmental, onion-skinlike distribution pattern similar to that in the nucleus caudalis.

\section{References}

1 Holtzman RN, Zablozki V, Yang WC, Leeds NE: Lateral pontine tegmental hemorrhage presenting as isolated trigeminal sensory neuropathy. Neurology 1987;37:704-706.
2 Kim JS, Lee MC, Kim HG, Suh DC: Isolated trigeminal sensory change due to pontine hemorrhage. Clin Neurol Neurosurg 1994; 96:168-169.

3 Komiyama M, Fu Y, Yagura H, Yasui T, Khosla VK, Berlit P: Pontine hemorrhages presenting as trigeminal neuropathy: report of three cases - Trigeminal neuropathy in pontine hemorrhage. Neurol Med Chir 1993;33: 234-237.

4 Almeida S, Chalk C, Minuk J, Del Carpio R, Guerin M, Levental M: Isolated trigeminal neuropathy due to trigeminal nerve root hemorrhage. Can J Neurol Sci 1999;26:204206.

5 Chung CS, Park CH: Primary pontine hemorrhage: a new CT classification. Neurology 1992;42:830-834.

6 Carpenter MB: Core Text of Neuroanatomy, ed 4. Baltimore, Williams \& Wilkins, 1991, pp 176-182.

7 Wall PD, Taub A: Four aspects of trigeminal nucleus and a paradox. J Neurophysiol 1962; 25:110-126.

8 Chiang CY, Hu B, Hu JW, Dostrovsky JO, Sessle BJ: Central sensitization of nociceptive neurons in trigeminal subnucleus oralis depends on integrity of subnucleus caudalis. J Neurophysiol 2002;88:256-264.

9 Goto F, Aamano T: Functional Neuroanatomy for Clinical Practice, ed 1. Tokyo, Chugai Igaku, 1992, pp 20-21. 